\title{
The effect of spatial structure in adaptive evolution
}

\author{
Lilia Perfeito ${ }^{1, *}$ Isabel Gordo ${ }^{1, *}$ Paulo Campos ${ }^{2, *}$ \\ ${ }^{1}$ Instituto Gulbenkian de Ciência, Rua Quinta Grande,6, Apartado 14, \\ P-2781-901 Oeiras, Portugal ${ }^{2}$ Universidade Federal Rural de Pernambuco, Brasil
}

\begin{abstract}
We study the dynamics of adaptation in a spatially structured population. The model assumes local competition for replication, where each organism interacts only with its nearest neighbors and is inspired by experimental methods that can be used to study the process of adaptive evolution in microbes. In such experiments microbial populations are grown on petri dishes and allowed to adapt by serial passage. We compare the rate of adaptation in a structured population where the structure is maintained intact to those where movement of individuals can occur. We observe that the rate of adaptive evolution is higher in intact structures than in structures with mixing. We have also studied the impact of structure on the distribution of adaptive mutations that fix during adaptation.
\end{abstract}

PACS numbers:

\section{Introduction}

Adaptation by Natural Selection is a very important aspect of the evolution of natural populations but it is still far from being completely understood. Early theoretical work tried to predict the fate of beneficial mutations in simple populations, particularly those with no structure. One of the most striking results of is that in finite populations, not all beneficial mutations will be fixed. More specifically, Haldane showed that when populations are large and the fitness advantage conferred by a new mutation is small, the probability that a beneficial mutation will be fixed is only twice its selective value [1]. His model was a very simple one where one locus with two alleles was the sole responsible for the fitness of an individual and it holds true for populations with sexual reproduction where each locus can be thought of as segregating independently of the rest due to frequent recombination.

As more loci are considered and if recombination is infrequent, the mutations can not be thought of as evolving independently. In fact, asexual populations are affected by the Hill Robertson effect, which states that selection at one locus reduces the efficacy of selection at a linked locus [2]. As a consequence of this effect, adaptation in asexual organisms has been shown to be strongly affected by the presence of deleterious mutations [3-6] and of other competing beneficial mutations segregating in the population [7-9]. This last type of interference was named clonal interference and has been shown theoretically and experimentally to limit the rate of adaptation of asexual populations [2,9-11]. The increase of the rate of fixation of beneficial mutations, slows down as the mutational input on the population $\left(N U_{b}\right)$ becomes very large. It has also been shown that clonal interference affects the distribution of mutations that get fixed: the bigger the mutational input, the more competition there is, so higher effect mutations get fixed [12]. Furthermore, the importance of this interference depends on the dele- terious mutation rate $[4,12]$.

The dynamics of adaptation has also been studied in structured populations where competition is local as opposed to the homogeneous model which assumes that every individual competes with all others. Indeed in natural populations competition is probably more commonly local. The dynamics of adaptation in such a population has been well established [13-18]. In particular, Maryuama, demonstrated that for some types of structure (such as the island model and other models that assume conservative migration) the same predictions hold as in the homogeneous population. But this is not valid when extinction and recolonization are allowed [19-21]. More recently, Gordo and Campos [22] have shown that when more than one locus is considered, clonal interference causes the structure to have a cost on adaptation, ie, the probability that a beneficial mutation is fixed decreases if there is structure and this decrease is bigger the higher the mutational input.

Here we extend on the model used in Gordo and Campos, relaxing the assumption that the structure remains intact as is probably the case for most natural populations. In particular, bacteria can form complex structures called biofilms, in which individuals may stay imprisoned in the biofilm matrix or have some degree of freedom to move [23] (and for a review see [24]). We modulate this movement by allowing mixing of the population to occur periodically. Competition remains local but now the neighbors are randomized after a given number of generations. We want to test how this type of mixing affects the adaptation of a population. This setup can be readily tested in experimental evolution with bacteria cultures growing on a solid medium which may be kept intact [25] with time or be completely randomized $[26,27]$. 


\section{The Model}

We consider a population of asexual haploid organisms. The individuals are arranged in a two-dimensional regular lattice of linear size $L$ and each organism occupies a cell in the lattice. The population size $N=L \times L$.

The evolution of the population follows a modified Wright-Fisher model to account for the chosen spatial structure. Each generation the individuals are all descendant from the previous one (non-overlapping generations) and competition is local. We study the Moore neighborhood where each individual competes with its eight nearest neighbors so that an organism occupying cell i can only be the descendant of individuals that occupied neighboring cells in the previous generation. The probability that an individual occupying cell i at generation $t+1$ is the offspring of the individual occupying cell $\mathrm{j}$ at $\mathrm{t}$ generation is given by:

$$
p_{i j}=\frac{\pi_{j}}{\sum_{l} \pi_{l}}
$$

where $\pi_{j}$ denotes the fitness value of individual $j$ and the sum is taken over cell $i$ and its eight neighbor sites. To model mutation events, we consider the infinite sites model. Each individual inherits all the mutations from its parent plus an additional number of deleterious mutations given by a Poisson with parameter $U$. Every deleterious mutation is assumed to decrease fitness by a constant factor $\left(1-s_{d}\right)$. Beneficial mutations occur at a constant rate $U_{b}$ per individual and they increase fitness by $\left(1+s_{b}\right)$. The $s_{b}$ for each beneficial mutation is taken from an exponential distribution with parameter $\beta$ [28-30].

$$
g\left(s_{b}\right)=\beta \exp \left(-\beta s_{b}\right)
$$

The fitness of each individual depends on the number of deleterious $(k)$ and beneficial $\left(k_{b}\right)$ mutations its genome has, such that its fitness is given by:

$$
\pi\left(k, k_{b}\right)=\left[\prod_{i=1}^{k_{b}}\left(1+s_{b}(i)\right)\right]\left(1-s_{d}\right)^{k} .
$$

At time $t=1$ all individuals are free of mutations. Then there is an additional equilibration period (of 100 generations) with no beneficial mutations being introduced. After this period we introduce one beneficial mutation in a randomly chosen individual. Subsequent advantageous mutations take place at a constant rate $U_{b}$ per individual. We have ascertained that our results do not change whether we consider a longer period of equilibration.

We assume that the individuals are mixed randomly in the population periodically. Every $T$ generations we randomly place the individuals in the lattice such that in the next generation they will have new competitors. By doing this we try to mimic the evolution experiment that can easily be done with bacteria in the laboratory, allowing in this way to directly test our results. When $T \rightarrow \infty$ then the structure of the population is intact and our results will be the same as those previously studied [22]. This way $T$ will modulate the randomness of the competition.

\section{Results}

\section{Probability of fixation of a beneficial mutation}

The rate of adaptation is affected by both the number of fixations as well as the distribution of mutations that become fixed. To understand how mixing of a spatially structured population affects its adaptation, we first ask whether the probability of fixation of a given beneficial mutation is affected by the periodicity of this mixing. In order to do this, we introduce a beneficial mutation with a given selective value $s_{b}$ at time $T=0$. After $T$ generations, the population is randomized so that each individual now competes with a different (and random) set of neighbors in the population. We let the simulation run until the beneficial mutation is either fixed or lost. Several simulations allow us to estimate the frequency of fixations. It was previously shown [22] that the probability of fixation of a beneficial mutation in this type of intact structure is the same as in a homogeneous population for this simple one locus model.

intuitively one could expect that, the more frequent mixing is, the more global competition will be and so the result would approach the homogeneous model. However, this is not the case. shows how mixing of the environment affects the probability of fixation of a beneficial mutation.

[b]

Probability of fixation of a beneficial mutation as a function of the periodicity of mixing $T$. In all simulations the population size was 2500 individuals.

As expected we see that when $T \rightarrow \infty$, the probability of fixation is approximately twice the selective value of the mutation. However, when mixing is frequent (for example when $T=10$ generations), the probability of fixation of a beneficial mutation becomes much smaller. Furthermore, the minimum value of $T$ above which we obtain the result for an homogeneous population depends on $s_{b}$ for $s_{b}=0.1$ it's approximately 50 but for $s_{b}=0.01$ it's about 200. This suggests that the frequency at which a mutation is, at the time of mixing, will influence its probability of fixation (see also [22]).

The probability that a beneficial mutation is lost due to drift depends on its frequency [? ]. In our model, when mixing occurs early after the appearance of the mutation, the mutant individuals will be scattered throughout the lattice. So locally, their frequency will become low and their probability of loss increases. This accounts for the 
fact that a low $T$ decreases greatly the probability of fixation. If enough generations pass before a shuffling occurs, the mutation will have the chance to grow in frequency (provided it escaped the initial stochastic loss) before it is scattered. This will increase the chance that individuals with beneficial mutations are grouped together and thus increases the probability of their fixation. The number of generations needed to reach this critical frequency will depend on the selective value of the mutation.

\section{Probability of fixation of a beneficial mutation}

As adaptation in asexuals may involve multiple loci, we have studied how the rate of fixation of beneficial mutations $\left(K_{b}\right)$ is affected by the frequency of mixing and the mutation rate $\left(U_{b}\right)$. If many mutations appear in the population per generation they will compete with each other and only the best will be fixed. This has two consequences; on one hand, after a certain value of $U_{b}$, $K_{b}$ does not increase. On the other, the selective value of the fixed mutations $\left(s_{b f i x}\right)$ increases. We have investigated how clonal interference is modulated by periodic mixing.[? ]summarizes the results.

[b]

Adaptation rate (a) and mean selective value of the fixed mutations (b) as a function of $T$. The data correspond to a set of 100 independent simulations ran over 100000 generations for the lower mutation rate and 10000 generations for the other values. The population size was 2500 individuals and beta was 100. No deleterious mutations were introduced

As expected from the results in, the adaptation rate increases with $T$. When mixing is frequent, most mutations are lost and those that get fixed have a high $s_{b}$ value ([?]). Again this suggests that the frequency that mutations reach at the time of mixing is critical to their fate. As a consequence, for large values of $T$, there are more fixations and the mean value of the mutations that become fixed decreases. In a sense, it becomes easier to fix a mutation if there is no mixing, even if it has a low selective effect.

Furthermore, above a certain value of $T, K_{b}$ and $s_{b f i x}$ become constant This critical value depends on the mean selective value of the newly arising mutations which suggests that when the mutations reach a certain frequency, mixing becomes irrelevant.

\section{Adaptation in the presence of deleterious mutations}

Since most newly arising mutations are deleterious, we next examined how they affect the dynamics of adaptation in our model. As previously shown for the intact structure, if the deleterious mutation rate $(U)$ is low, which implies that the mean number of deleterious mutations is small, they have very little effect on $K_{b}$ and on $s_{\text {f fix }}$. If, however, their rate of appearance is high, they decrease the adaptation rate [22].

In Figure 1 we show how deleterious mutations affect both $K_{b}$ and $s_{b f i x}$. From the Figure we observe that $K_{b}$ becomes less dependent on $T$, when deleterious mutations are present.

The reduction in $K_{b}$ reflects the reduction in the proportion of mutation-free genotypes when $U \neq 0$. This can be interpreted as a lowering of the effective population size, so fewer adaptive mutations are fixed [3].

Figure $3 \mathrm{~b}$ shows that If mixing is frequent, the mean selective value of fixed mutations is not affected by the presence of deleterious mutations (which wouldn't be expected to change if only the effective size of the population decreases). However, as mixing becomes less frequent, the mean effect of mutations that get fixed is reduced (Figure 3b). It is in this regime that clonal interference plays a more important part because more mutations are segregating and fixing in the population. Deleterious mutations tend to make this interference less important [? ] and that explains why the fitness effect of fixed beneficial mutations is lower if there are deleterious mutations than in their absence.

\section{Discussion}

We have studied a population with a simple spatial structure where the individuals are arranged in a regular lattice and competition is local. In order to study the impact of mixing on the adaptation of such a population, we periodically randomized the neighbors so that each individual will now compete with a different set of competitors than his ancestors did. Unlike what one could expect, this did not approximate the results for an homogeneous population where competition is global. In fact, the more frequently we randomize the network the further away we get from the rate of adaptation in a homogeneous population. This is due to the fact that even though the competitors could be any in the population, competition is still local at any given time point. Furthermore, by scattering a beneficial mutation shortly after its appearance, we are increasing genetic drift and thus lowering its chances of survival. Most natural populations have some sort of spatial structure. So even though the predictions of the models with global competition have been proven valid in a number of experiments, other fac-

FIG. 1: Adaptation rate (a) and mean selective value of the fixed mutations (b) as a function of $T$. The data correspond to a set of 100 independent simulations ran over 100000 generations. The population size was 2500 individuals and beta was 100. The beneficial mutation rate was 0.0001 and $s d=0.1$ 
tors must be taken into account when studying natural populations. Namely we see a clear effect of global randomization on the adaptation rate. In addition, this regime may be a good way to modulate adaptation within bacterial biofilms, where different levels of movement are observed. In laboratory conditions, bacteria can grow on solid medium, thus competing only locally and these cultures may be propagated maintaining the structural integrity or by randomizing them periodically. So our results can be tested and help us understand how adaptation occurs in natural populations.

This work was supported by project POCTI/BSE/46856/2002 through Fund. para a Ciência e Tecnologia (FCT). L. Perfeito and I. Gordo were supported by FCT fellowships SFRH/BD/18161/2004 and SFRH/BPD/8104/2002.P.C. is partially supported by Conselho Nacional de Desenvolvimento Científico e Tecnológico (CNPq).

[1] Haldane, J. B. S. (1927). A mathematical theory of natural and artificial selection. part v: Selection and mutation. Proc. Camb. Phil. Soc. 26, 220230

[2] Hill, W. G. and A. Robertson (1966). The effect of linkage on the limits to artificial selection. Genet. Res. 8, 269294.

[3] Charlesworth, B., M. Morgan, and D. Charlesworth (1993). The effect of deleterious mutations on neutral molecular variation. Genetics 134, 12891303.

[4] Orr, H. A. (2000). The rate of adaptation in asexuals. Genetics 155, 961968.

[5] Bachtrog, D. and I. Gordo (2004). Adaptive evolution of asexual populations under Muller's ratchet. Evolution 58, 14031413.

[6] Wilke, C. O. (2004). The speed of adaptation in large asexual populations. Genetics 167, 20452053.

[7] Barton, N. H. (1994). The reduction in fixation probability caused by substitutions at linked loci. Genetical Research 64, 199208.

[8] Barton, N. H. (1995). Linkage and the limits to natural selection. Genetics 140, 821841.

[9] Gerrish, P. J. and R. E. Lenski (1998). The fate of competing beneficial mutations in an asexual population. Genetica 102, 127144.

[10] Miralles R, Gerrish PJ, Moya A, Elena SF.(1999) Clonal interference and the evolution of RNA viruses.Science 10;285(5434):1745-7.

[11] Arjan JA, Visser M, Zeyl CW, Gerrish PJ, Blanchard JL, Lenski RE. (1999) Diminishing returns from mutation supply rate in asexual populations. Science. 15;283(5400):404-6.

[12] de Oliveira, V. M. and P. R. A. Campos (2004). Dynamics of fixation of advantageous mutations. Physica A 337,
546554 .

[13] Wright, S. (1931). Evolution in mendelian populations. Genetics 16, 97159.

[14] Maruyama, T. (1970). On the probability of fixation of mutatnt genes in subdivided populations. Genetical Research 15, 221225.

[15] Maruyama, T. (1974). A simple proof that certain quantities are independent of the geographical structure of population. Theoretical Population Biology 5, 148154.

[16] Slatkin, M. (1981). Fixation probabilities and times in a subdivided population. Evolution 35, 477488.

[17] Nagylaki, T. (1980). The strong-migration limit in geographically populations. Journal of Mathematical Biology $9,101114$.

[18] Nagylaki, T. (1982). Geographical invariance in population genetics. Journal of Theoretical Biology 99, 159172.

[19] Barton, N. (1993). The probability of fixation of a favourable allele in a subdivided population. Genetical Research 62, 149158.

[20] Whitlock, M. C. (2002). Selection, load and inbreeding depression in a large metapopulation. Genetics 160, 11911202.

[21] Rose, D. and F. Rousset (2003). Selection and drift in subdivided populations: a straightforward method for deriving diffusion approximations and applications involving dominace, selfing and local extintions. Genetics 165, 21532166

[22] Gordo, I and Campos, P (2005) Adaptive evolution in a spatially structured population. Genetica

[23] Rice AR, Hamilton MA, Camper AK (2003) Movement, replication, and emigration rates of individual bacteria in a biofilm. Microbial Ecology 45(2):163-72. Epub 2002 Dec 20

[24] Costerton JW, Stewart PS, Greenberg EP (1999)Bacterial Biofilms: A Common Cause of Persistent infections Science 284, 1318

[25] Angus Buckling, Rees Kassen, Graham Bell, Paul B.Rainey Disturbance and diversity in experimental microcosms. Nature 408, 961-964 (21 Dec 2000)

[26] F., Conceio, I.C., Marques A.C.R., Fernandes, L. and Gordo, I. 2005. The evolution of a conjugative plasmid and its ability to increase bacterial fitness Biol. Lett. 1:250-252.

[27] Paul B. Rainey, Michael Travisano Adaptive radiation in a heterogeneous environment Nature 394, 69-72 (02 Jul 1998) Letters to Editor

[28] Gillespie, J. H. (1991). The Causes of Molecular Evolution. Oxford University Press.

[29] Orr, H. A. (2003). The distribution of fitness effects among beneficial mutations. Genetics 163, 15191526.

[30] Rozen, D. E., J. A. G. M. de Visser, and P. J. Gerrish (2002). Fitness effects of fixed beneficial mutations in microbial populations. Curr. Biol. 12, 10401045.

[31] Mathematical Population Genetics Springer 